\title{
Discussion
}

\section{Optimising Results from Minimal Access Cranial Suspension Lifting}

\author{
Yann Levet \\ Boulogne, France
}

Over the past 40 years, about 1,000 rejuvenating surgical techniques to correct the aging face have been described. The methods that have survived the test of time to be used in everyday practice are efficient, logical, and reliable, with few complications. In fact, many techniques are good, but a technique is nothing if it is not adapted to a particular case. We must adapt good methods to good patients. In other words, we must identify the indications, so we must be aware of all the procedures.

As I said in a recent ISAPS meeting, my technique for the middle face is the result of many procedures taken from almost 10 different surgeons. The precapillary incision I sometimes use was described by B. Connell, and the preauricular incision is from I. Pitanguy. Another technique I use inside the sideburn was performed by B. Guyuron. My retroauricular incision was described by S. Hoefflin, and my primary liposuction of the neck was used by Y. Illouz. The skin undermining I have adopted was reported by Skoog, Aufricht, and Mac Indoe, and my deep muscular plane treatment is the one used by G. Jost and me. Often I use a deep undermining of the fat to suspend the malar pad, as described by S. Hamra, and I often add a transconjunctival approach associated with light erbium laser abrasion of the inferior eyelid skin, as advocated by T. Roberts. I could add that I suspend the platysma angle without undermining the neck skin, as done by D. Labbe, and I could also use a vertical purse suture, as shown by P. Tonnard. Why not? It is also an effective technique when appropriate.

In fact, these are the logical findings by the authors of this article. Platysma suspension by a purse suture to the deep plane or to the Lore's fascia is not sufficient in the presence of significant anterior platysma bands. Therefore, it is logical in these cases to include an anterior cervicoplasty. The vertical suspension of a minimal access cranial suspension (MACS) lift cannot deal with excess posterior skin on the neck. Therefore, in these cases, a posterior cervicoplasty is also mandatory.

Consequently, we must adapt our techniques to the patient, to her or his skin conditions, hair situation, former operations, shape of the platysma on the neck, particular excess of skin, and the like. One single procedure is not sufficient to please the patient, and one technique is never completely adapted to one case. We always need additional procedures for the finesse of a result. The authors of the article have shown this perfectly. 